\title{
The phase diagram of $\mathrm{K}_{0.5} \mathrm{Na}_{0.5} \mathrm{NbO}_{3}-\mathrm{Bi}_{1 / 2} \mathrm{Na}_{1 / 2} \mathrm{TiO}_{3}$
}

\section{Laijun Liu, Michael Knapp, Helmut Ehrenberg, Liang Fang, Ljubomira Ana Schmitt, Hartmut Fuess, Markus Hoelzel and Manuel Hinterstein}

J. Appl. Cryst. (2016). 49, 574-584

\section{IUCr Journals CRYSTALLOGRAPHY JOURNALS ONLINE}

Copyright (C) International Union of Crystallography

Author(s) of this paper may load this reprint on their own web site or institutional repository provided that this cover page is retained. Republication of this article or its storage in electronic databases other than as specified above is not permitted without prior permission in writing from the IUCr.

For further information see http://journals.iucr.org/services/authorrights.html 


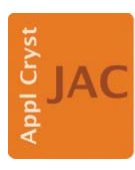

ISSN 1600-5767

Received 30 July 2015

Accepted 17 February 2016

Edited by J. Hajdu, Uppsala University, Sweden

Keywords: $\mathrm{K}_{0.5} \mathrm{Na}_{0.5} \mathrm{NbO}_{3} ; \mathrm{Bi}_{1 / 2} \mathrm{Na}_{1 / 2} \mathrm{TiO}_{3}$; phase diagrams; morphotropic phase boundaries; polymorphic phase boundaries.

Supporting information: this article has supporting information at journals.iucr.org/j

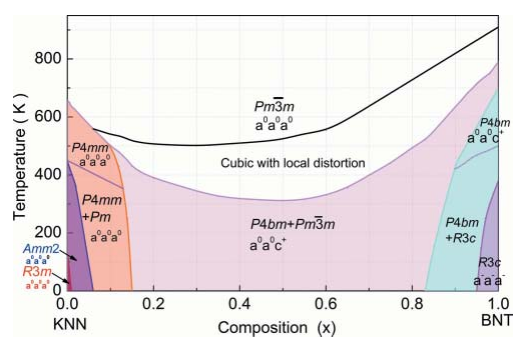

C 2016 International Union of Crystallography

\section{The phase diagram of $\mathrm{K}_{0.5} \mathrm{Na}_{0.5} \mathrm{NbO}_{3}-$ $\mathrm{Bi}_{1 / 2} \mathrm{Na}_{1 / 2} \mathrm{TiO}_{3}$}

\author{
Laijun Liu, ${ }^{\mathrm{a}, \mathrm{b} *}$ Michael Knapp, ${ }^{\mathrm{a}}$ Helmut Ehrenberg, ${ }^{\mathrm{a}}$ Liang Fang, ${ }^{\mathrm{b}}$ Ljubomira Ana \\ Schmitt, ${ }^{\mathrm{c}}$ Hartmut Fuess, ${ }^{\mathrm{c}}$ Markus Hoelzel ${ }^{\mathrm{d}}$ and Manuel Hinterstein ${ }^{\mathrm{a}, \mathrm{e}}$
}

anstitute for Applied Materials (IAM), Karlsruhe Institute of Technology (KIT), Hermann-von-Helmholtz-Platz 1, Eggenstein-Leopoldshafen D-76344, Germany, ${ }^{\mathbf{b}}$ Key Laboratory of New Processing Technology for Non-ferrous Metals and Materials of Ministry of Education of China, College of Materials Science and Engineering, Guilin University of Technology, Guilin 541004, People's Republic of China, 'Institute of Materials Science, Technische Universität Darmstadt, D-64287, Germany, ${ }^{\mathbf{d}}$ Forschungsneutronenquelle Heinz Maier-Leibnitz (FRM II), Technische Universität München, Lichtenbergstrasse 1, Garching D-85747, Germany, and ${ }^{\mathbf{e}}$ School of Materials Science and Engineering, UNSW Australia, Sydney 2052, Australia. *Correspondence e-mail: ljliu2@163.com

The phase diagram of the lead-free piezoelectric $(1-x) \mathrm{K}_{0.5} \mathrm{Na}_{0.5} \mathrm{NbO}_{3}-$ $x \mathrm{Bi}_{1 / 2} \mathrm{Na}_{1 / 2} \mathrm{TiO}_{3}$ system has been studied by high-resolution synchrotron powder diffraction, neutron powder diffraction and selected area electron diffraction. The two lead-free piezoelectric materials $\mathrm{K}_{0.5} \mathrm{Na}_{0.5} \mathrm{NbO}_{3}$ and $\mathrm{Bi}_{1 / 2} \mathrm{Na}_{1 / 2} \mathrm{TiO}_{3}$ form an infinite substitution solid solution. The orthorhombic $(O)$, monoclinic $(M)$, tetragonal $(T)$ and rhombohedral $(R)$ phases and the phase coexistence of $M(P m)+T(P 4 m m)$ for $0.02<x \leq 0.14, T(P 4 b m)+$ pseudocubic $(P m \overline{3} m)$ for $0.14<x \leq 0.87$ and $T(P 4 b m)+R(R 3 c)$ for $0.87<x \leq 0.96$ have been investigated at room temperature, with a subtle change in the structure observed. The oxygen octahedral tilt system has been mapped as a function of composition and temperature. The results indicate that $\mathrm{K}_{0.5} \mathrm{Na}_{0.5} \mathrm{NbO}_{3}-$ $\mathrm{Bi}_{1 / 2} \mathrm{Na}_{1 / 2} \mathrm{TiO}_{3}$ does not display a morphotropic phase boundary like lead zirconate titanate, and that the most significant structural changes as a function of composition occur near $x=0.14$ and $x=0.87$ as a result of ionic disorder at the $A$ and $B$ sites in the perovskite $A B \mathrm{O}_{3}$ structure at room temperature.

\section{Introduction}

The development of lead-free replacements for the archetypal piezoelectric material $\mathrm{PbZr}_{x} \mathrm{Ti}_{1-x} \mathrm{O}_{3}$ (PZT) has received much attention because of environmental concerns over lead being released into the atmosphere during processing. Potassium sodium niobate $\left(\mathrm{K}_{0.5} \mathrm{Na}_{0.5} \mathrm{NbO}_{3}\right)$ and bismuth sodium titanate $\left(\mathrm{Bi}_{1 / 25} \mathrm{Na}_{1 / 2} \mathrm{TiO}_{3}\right)$ are considered as a good replacement for PZT because of their good piezoelectric properties (Shrout \& Zhang, 2007; Takenaka et al., 2008; Panda, 2009; Rödel et al., 2009). $\mathrm{K}_{0.5} \mathrm{Na}_{0.5} \mathrm{NbO}_{3}$ (KNN) has cubic symmetry in space group $P m \overline{3} m$ above $690 \mathrm{~K}$ and tetragonal symmetry $(P 4 m m)$ in the temperature range $470-690 \mathrm{~K}$. When $\mathrm{KNN}$ is cooled below $470 \mathrm{~K}$, the tetragonal phase transforms to an orthorhombic phase $(A m m 2)$, which in turn transforms at about $110 \mathrm{~K}$ to a rhombohedral phase $(R 3 m)$ (Tellier et al., 2009). The structural changes of $\mathrm{Bi}_{1 / 2} \mathrm{Na}_{1 / 2} \mathrm{TiO}_{3}$ (BNT) with temperature are even more complex. Jones \& Thomas (2002) found two temperature-dependent phase transitions in the temperature range $573-593 \mathrm{~K}$, from the low-temperature ferroelectric phase in space group $R 3 c$ to the high-temperature phase in space group $P 4 \mathrm{bm}$. The ferroelectric to paraelectric phase transition was assigned to $813 \mathrm{~K}$. The octahedral tilting of the two polar ferroelectric phases can be described by the Glazer (1975) notation as $a^{-} a^{-} a^{-}$for the $R 3 c$ phase and $a^{0} a^{0} c^{+}$for the $P 4 b m$ phase. However, according to the 
dielectric and birefringence properties, three phase transitions associated with four different phases are found in the overall temperature range. With increasing temperature, a phase transition from ferroelectric (rhombohedral) to antiferroelectric (rhombohedral + tetragonal) occurs at $\sim 520 \mathrm{~K}$ (Jones \& Thomas, 2002), corresponding to roughly the lowtemperature anomaly. Then the antiferroelectric phase changes into a weakly polar (or nonpolar) phase (tetragonal) (Jones \& Thomas, 2000) with a main dielectric anomaly at $\sim 700 \mathrm{~K}$, where the Curie-Weiss law is fulfilled and BNT is clearly paraelectric. Finally, the cubic aristotype $(\operatorname{Pm} \overline{3} m)$ appears at about $800 \mathrm{~K}$ up to the melting point at $1563 \mathrm{~K}$ (Dorcet et al., 2008). However, the paraelectric phase is stable below $700 \mathrm{~K}$, as the main dielectric anomaly at about $593 \mathrm{~K}$ represents the change from the antiferroelectric to the paraelectric behavior.

Recently, there has been some debate over the structural transitions of BNT with changes in temperature. A monoclinic space group $C c$ was found to better describe the average structure of BNT single crystals (Gorfman \& Thomas, 2010; Gorfman et al., 2012) and powder (Aksel et al., 2011) at room temperature. For the powder sample, the $C c$ space group is only revealed in sintered powder, while calcined powder is equally fitted by an $R 3 c$ model. BNT exhibits two anomalies in the temperature dependence of the dielectric permittivity. Peculiar propeller-shaped hysteresis loops have often been found at $\sim 520 \mathrm{~K}$ in BNT, which could be considered as the appearance of the antiferroelectric phase. However, detailed transmission electron microscopy (TEM) studies suggest a much more complex local structure or low-symmetry average structure in BNT-based compositions (Trolliard \& Dorcet, 2008; Levin \& Reaney, 2012). In fact, the occurrence of the double loop can be induced by various mechanisms.

Good piezoelectric properties are observed for solid solutions within a transition region of the phase diagrams known as the morphotropic phase boundary (MPB; Jaffe et al., 1971), where the crystal structure changes suddenly with composition. However, KNN often exhibits a diffuse polymorphic phase transition between orthorhombic and tetragonal phases when the temperature is lowered to near room temperature (Fu et al., 2010; Liu et al., 2012), which results in a polymorphic phase boundary (PPB). Unlike an MPB located in a narrow composition region and almost temperature independent, as in the PZT system, the position of the PPB in most KNN solid solutions shifts gradually and continuously to lower temperatures (Zhang et al., 2007; Shrout \& Zhang, 2007; Dai et $a l ., 2007)$. For example, in $(1-x) \mathrm{KNN}-x M \mathrm{TiO} 3(M=\mathrm{Pb}, \mathrm{Ba}$, $\mathrm{Sr}$ and $\mathrm{Ca}$ ) solid solutions, both the Curie temperature $T_{\mathrm{C}}$ and the tetragonal-orthorhombic phase transition temperature $T_{T-O}$ decrease sharply at low $x$ range and are almost independent of $M$ (Wang et al., 2009). The PPB is quite similar to the frequently encountered MPB as it enables phase coexistence, but the crystal structure of the compositions near the PPB is more sensitive to temperature. An MPB describes a symmetry change as a function of composition, while a PPB describes a symmetry change as a function of temperature and/or composition. However, the mechanism concerning how the phase coexistence enhances piezoelectric properties in both cases looks similar and is probably the result of different orientations of the spontaneous polarization vectors within the slightly different crystal structures and thereby an increased strain effect in applied electrical fields. In contrast to the PZT system, no MPB has been found in the KNN-BNT system. Zuo et al. (2007) found that $(1-x) \mathrm{KNN}-x \mathrm{BNT}$ exhibits orthorhombic $(x \leq 0.02)$, tetragonal $(0.03 \leq x \leq 0.09)$, cubic $(0.09<x \leq 0.20)$ and rhombohedral $(x>0.20)$ symmetries at room temperature. An MPB between orthorhombic and tetragonal ferroelectric phases was reported in the composition range of $0.02<x<0.03$ (Zuo et al., 2007), but later interpreted as a 'PPB' because of the strong temperature dependence of the corresponding phase transition (Du et al., 2008). Kounga and co-workers studied the electromechanical behavior of $(1-x) \mathrm{KNN}-x \mathrm{BNT}$ ceramics for the BNT-rich compositions $0.88 \leq x \leq 1.00$ to gain insight into the giant strain on the basis of an antiferroelectric-ferroelectric (AFEFE) phase transition. An MPB between a rhombohedral FE phase and a tetragonal AFE phase at $x \simeq 0.93$ was concluded from the observed giant strain (Kounga et al., 2008). However, the underlying crystal structures in the KNN-BNT system are essentially unknown, because deviations from the cubic like phase are difficult to detect by X-ray diffraction. In this paper, a comprehensive study of the $(1-x) \mathrm{KNN}-x \mathrm{BNT}$ phase diagram is reported. Complementary synchrotron, neutron and electron diffraction analyses were employed to reveal the structure evolution of the solid solution system. Besides the above-mentioned phase boundaries, two more phase boundaries between structures with different oxygen octahedral tilt systems are found near $x \simeq 0.14$ and $x \simeq 0.87$ at room temperature, which might lead to remarkable physical properties in this composition range.

\section{Experimental}

\subsection{Sample preparation}

Samples of $(1-x) \mathrm{KNN}-x \mathrm{BNT}(x=0.00,0.005,0.02,0.04$, $0.06,0.10,0.20,0.30,0.50,0.70,0.90,0.92,0.94,0.96,0.98,1.00)$ were prepared by a solid state reaction. Highly pure carbonates and oxides $\mathrm{K}_{2} \mathrm{CO}_{3} \cdot 1.5 \mathrm{H}_{2} \mathrm{O}$ (MERCK, 99.995\%), $\mathrm{Na}_{2} \mathrm{CO}_{3}$ (PROLABO, 99.9\%), $\mathrm{Nb}_{2} \mathrm{O}_{5}$ (INTERCHIM, 99.5\%), $\mathrm{Bi}_{2} \mathrm{O}_{3}$, (Alfa Aesar, 99.975\%) and $\mathrm{TiO}_{2}$ (Aldrich, 99.8\%) were used as starting materials. In all the experiments, the raw materials were dried before use at $423 \mathrm{~K}$ for $12 \mathrm{~h}$ because of their hygroscopic nature. The powders were weighed according to the stoichiometric formula. For the samples used in dielectric, $\mathrm{X}$-ray diffraction and electron diffraction measurements, the raw materials were mixed in a planetary mill (RETSCH PM 100) at $450 \mathrm{r} \mathrm{min}^{-1}$ for $8 \mathrm{~h}$ (Liu et al., 2011, 2013). For the samples used in neutron diffraction measurement, the powders were milled in ethanol with the high-energy ball milling technique (FRITSCH Pulverisette 7), at a rotational speed of $800 \mathrm{rmin}^{-1}$ for $1 \mathrm{~h}$. The mixed powders were calcined at $1123 \mathrm{~K}$ for $2.5 \mathrm{~h}$ and then ball milled again for $2 \mathrm{~h}$. The resulting powder was uniaxially pressed into discs of 
$10 \mathrm{~mm}$ in diameter and $2 \mathrm{~mm}$ in thickness under $300 \mathrm{MPa}$ and then pressed under $650 \mathrm{MPa}$ with the cool isostatic pressing method. The samples for neutron diffraction were pressed into cylinders with the different size of $13 \mathrm{~mm}$ diameter and $10 \mathrm{~mm}$ in height. These discs were sintered in air for $2 \mathrm{~h}$ in a sealed alumina crucible. The sintering temperature was increased from 1343 to $1473 \mathrm{~K}$ with increasing BNT content.

\subsection{Powder diffraction}

All high-resolution synchrotron measurements were carried out at the powder diffraction beamline P02.1 at PETRA III (DESY, Hamburg, Germany) (Herklotz et al., 2013). The beamline operates at a fixed energy of approximately $60 \mathrm{keV}$. The wavelength was determined to be 0.20724 (5) A by using an $\mathrm{LaB}_{6}$ NIST standard. Neutron diffraction measurements were performed at the SPODI powder diffractometer at the research reactor FRM-II (MLZ, Garching, Germany) at an incident wavelength of $1.5484 \AA$ (Hoelzel et al., 2012). Data were collected by a bank of 80 position-sensitive ${ }^{3} \mathrm{He}$ detectors, covering a $160^{\circ}$ scattering range.

Full-profile Rietveld refinements were performed using the software package FULLPROF (Roisnel \& RodriguezCarvajal, 2001). The peak profile shape was described by a pseudo-Voigt function (Thompson et al., 1987). The background of the diffraction patterns was fitted using linear interpolation between selected data points in non-overlapping regions. The scale factor, zero angular shift, lattice parameters, atomic positions, isotropic displacement parameters and profile shape parameters were varied during the refinement.

\subsection{Transmission electron microscopy}

For TEM observation, samples were prepared by a standard procedure of polishing, disc cutting, dimpling and ion thinning. Thin slices were polished down to approximately $120 \mu \mathrm{m}$ in thickness and ultrasonically cut into discs of $\sim 3 \mathrm{~mm}$ in diameter. The discs were mechanically dimpled and finally thinned on both sides by an $\mathrm{Ar}^{+}$ion beam. Specimens were lightly coated with a thin carbon coat to prevent charging under the incident electron beam. TEM was performed on a CM20 (FEI, Eindhoven, The Netherlands) instrument operating at $200 \mathrm{kV}$. The orientation matrices of the grains were directly determined by a program for interpreting electron diffraction patterns (PIEP; Miehe, 2002). Zone axes were indexed according to the cubic aristotype structure. Representative grains were examined on the $[001]_{C},[011]_{C}$ and $[013]_{\mathrm{C}}$ zone axes.

\section{Results and discussion}

3.1. Powder diffraction: crystal structure as a function of composition

Fig. 1 shows synchrotron diffraction (SD) patterns of $(1-x) \mathrm{KNN}-x \mathrm{BNT}$ in the composition range of $0.00 \leq x \leq$ 1.00 with a logarithmic scale for the intensity. Throughout this work, the $h k l$ indices are defined with respect to the idealized pseudocubic coordinate reference frame for consistency in spite of the different space groups resulting from symmetry reduction by small distortions and octahedral tilts. By consideration of the SD patterns in combination with dielectric and Raman spectroscopy data (Liu et al., 2015), the patterns could be distinguished into five composition ranges on the basis of the Bragg reflections and their profiles. The Bragg profiles of $x \leq 0.005$ are similar to that of pure KNN, which indicates this region, denoted as Region (I), as a solid solution with the same crystal structure. With the increase of BNT content, drastic changes become visible in the range of $0.02<x \leq 0.10$, which is denoted as Region (II). All Bragg reflections of compositions within Region (III), $0.20 \leq x \leq$ 0.50 , match with a pseudocubic perovskite structure. With a further increase of the BNT concentration to $0.90 \leq x$, reflections characteristic of the out-of-phase octahedral tilt $\frac{1}{2} 311$ (marked by an arrow in Fig. 1) become visible and more intense, indicating the appearance of a rhombohedral structure. However, the range of $0.90 \leq x \leq 1.00$ should include two regions, because an AFE-FE phase transition occurs at $x \simeq$ 0.95 (Kounga et al., 2008). Therefore, $0.90 \leq x \leq 0.96$ is denoted as Region (IV) and $0.96<x \leq 1.00$ as Region V. It is noted that two impurities, $\mathrm{K}_{6} \mathrm{Nb}_{10.88} \mathrm{O}_{30}$ (for $0 \leq x \leq 0.06$ ) and $\mathrm{KTiNbO}_{5}$ (for $0.10 \leq x \leq 0.20$ ), can be found in the $\mathrm{X}$-ray diffraction patterns, which might result from $\mathrm{Na} / \mathrm{K} / \mathrm{Bi}$ evaporation. Since the fraction of impurities is very small, no obvious stoichiometric change of the samples is found according to the Rietveld refinement results. The details of symmetry and structure for different compositions are discussed in the next sections.

3.1.1. Rietveld analysis of powder diffraction data for $0 \leqq$ $x \leq 0.005$. The synchrotron powder diffraction patterns at room temperature were analyzed on the basis of an orthorhombic perovskite structure with the space group Amm2. Variations in reflection widths, especially for the $002_{\mathrm{C}}$ and $200_{C}$ reflections, could be modeled with an anisotropic broadening model (Stephens, 1999). The detailed structural

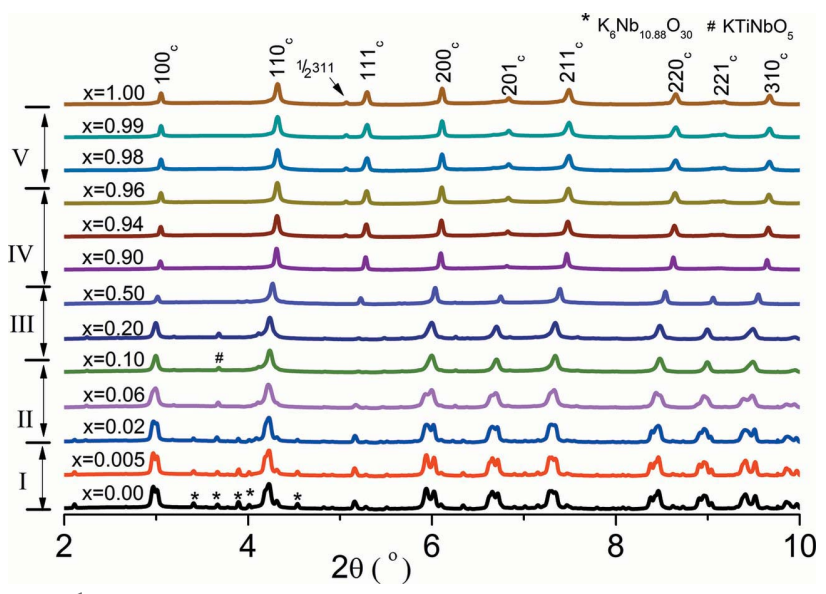

Figure 1

Evolution of synchrotron powder diffraction patterns of $(1-x) \mathrm{KNN}-$ $x$ BNT ceramics with composition $(x)$ at room temperature with a logarithmic scale for the intensity. The Miller indices are with respect to the pseudocubic perovskite cell. The phase structure could be divided into five regions on the basis of dielectric and Raman data and splitting of Bragg reflections. Superlattice reflections $\frac{1}{2} 311$ are marked by an arrow. 
Table 1

Structural parameters, fractional atomic coordinates and equivalent isotropic displacement parameters $\left(B_{\text {iso }}\right.$ in $\AA^{2}$ ) from the SD refinements of the samples of KNN-0.00BNT and KNN-0.005BNT.

\begin{tabular}{|c|c|c|c|c|}
\hline \multirow{3}{*}{$\begin{array}{l}x=0.00 \\
\begin{array}{l}a, b, c(\AA) \\
\alpha, \beta, \gamma\left({ }^{\circ}\right)\end{array}\end{array}$} & \multicolumn{4}{|c|}{ Orthorhombic phase $(A m m 2)$} \\
\hline & $3.9455(6)$ & $5.6419(1)$ & $5.6728(3)$ & \\
\hline & 90 & 90 & 90 & \\
\hline $\mathrm{Na} / \mathrm{K} x, y, z, B_{\text {iso }}$ & 0 & 0 & 0 & $1.0064(4)$ \\
\hline $\mathrm{Nb} x, y, z, B_{\text {iso }}$ & $1 / 2$ & 0 & $0.4873(3)$ & $0.0825(2)$ \\
\hline O1 $x, y, z, B_{\text {iso }}$ & $1 / 2$ & $1 / 4$ & $0.2598(3)$ & $0.7733(9)$ \\
\hline $\mathrm{O} 2 x, y, z, B_{\text {iso }}$ & 0 & 0 & $0.5286(9)$ & $0.1172(6)$ \\
\hline$R_{\mathrm{p}}, R_{\mathrm{wp}}, R_{\mathrm{e}}, \chi^{2}$ & 5.95 & 8.07 & 2.70 & 8.921 \\
\hline$x=0.005$ & \multicolumn{4}{|c|}{ Orthorhombic phase $(A m m 2)$} \\
\hline$a, b, c(\AA)$ & $3.9453(4)$ & $5.6412(2)$ & $5.6721(2)$ & \\
\hline$\alpha, \beta, \gamma\left({ }^{\circ}\right)$ & 90 & 90 & 90 & \\
\hline $\mathrm{Na} / \mathrm{K} / \mathrm{Bi} x, y, z, B_{\text {iso }}$ & 0 & 0 & 0 & $1.5067(8)$ \\
\hline $\mathrm{Nb} / \mathrm{Ti} x, y, z, B_{\text {iso }}$ & $1 / 2$ & 0 & $0.5117(8)$ & $0.2808(1)$ \\
\hline $\mathrm{O} 1 x, y, z, B_{\text {iso }}$ & $1 / 2$ & $1 / 4$ & $0.2968(8)$ & $1.0675(6)$ \\
\hline $\mathrm{O} 2 x, y, z, B_{\text {iso }}$ & 0 & 0 & $0.5508(3)$ & $0.6036(7)$ \\
\hline$R_{\mathrm{p}}, R_{\mathrm{wp}}, R_{\mathrm{e}}, \chi^{2}$ & 6.54 & 8.01 & 2.67 & 9.003 \\
\hline
\end{tabular}

Phase fraction of impurity $\mathrm{K}_{6} \mathrm{Nb}_{10.88} \mathrm{O}_{30}$ is slightly below $2 \%$ for $x=0.00$ and $x=0.005$.

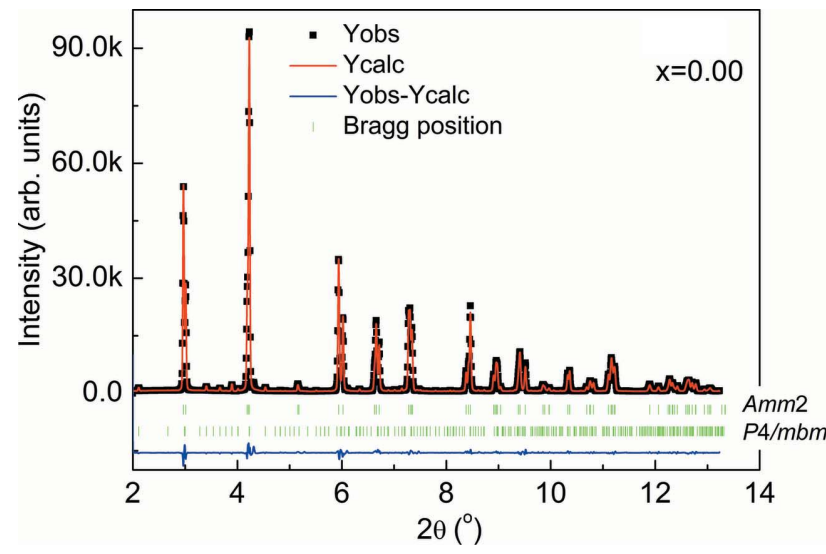

(a)

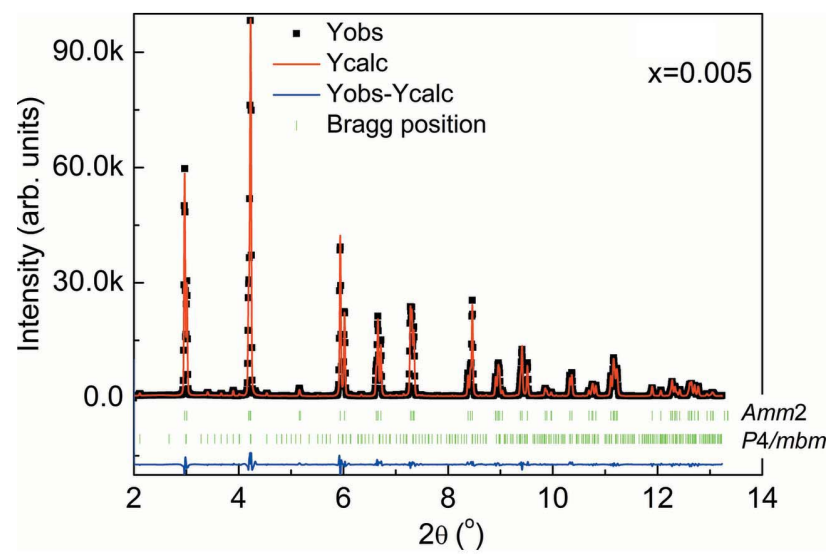

(b)

Figure 2

Rietveld refinements based on synchrotron diffraction data of $(a) \mathrm{KNN}-$ $0.00 \mathrm{BNT}$ and $(b) \mathrm{KNN}-0.005 \mathrm{BNT}$. parameters and goodness of fit for the orthorhombic phase of $(1-x) \mathrm{KNN}-x \mathrm{BNT}$ with $x=0.00$ and $x=0.005$, as obtained from the SD data, are given in Table 1 . The fit between the observed and calculated profiles is satisfactory, as shown in Fig. 2(a) for $x=0.00$ and Fig. 2(b) for $x=0.005$. Both samples include traces of a $\mathrm{K}_{6} \mathrm{Nb}_{10.88} \mathrm{O}_{30}$ impurity, $\sim 2 \%$. In accordance

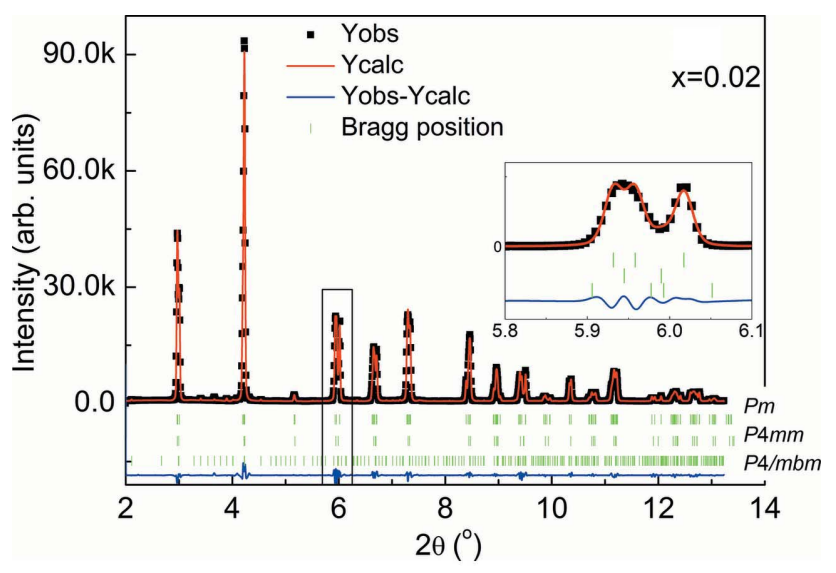

(a)

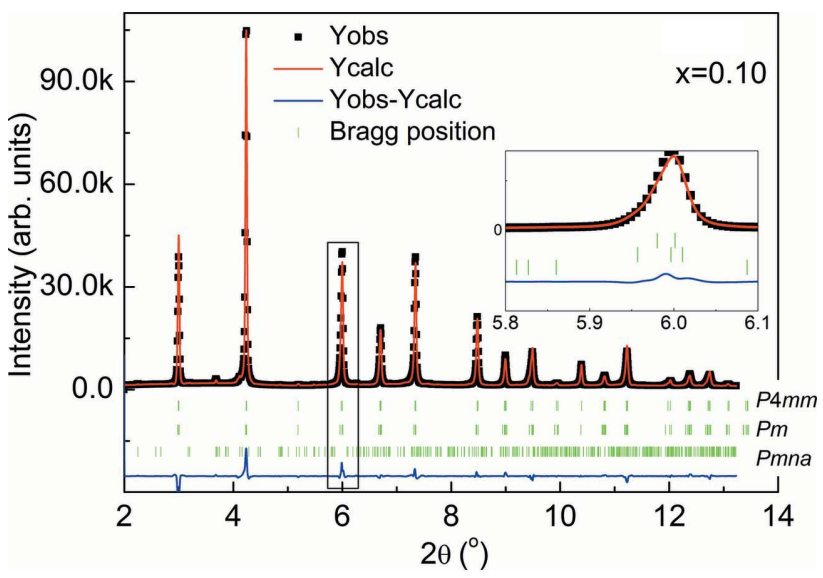

(b)

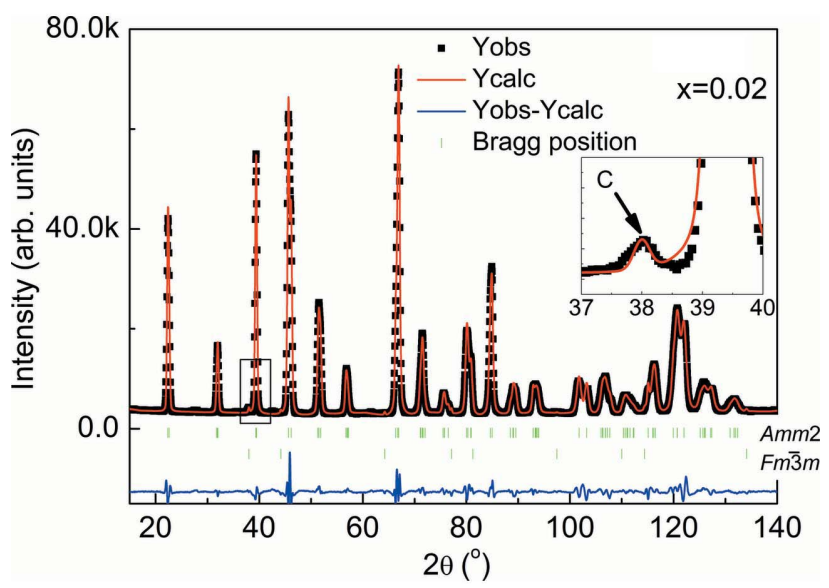

(c)

Figure 3

Rietveld refinements based on synchrotron diffraction data of $(a) \mathrm{KNN}-$ $0.02 \mathrm{BNT}$ and $(b) \mathrm{KNN}-0.10 \mathrm{BNT}$. Insets show the regions around the 200 reflection. (c) Similar, but for neutron diffraction data of KNN-0.02BNT at $4 \mathrm{~K}$. The peak at $2 \theta=38.1^{\circ}$ marked with ' $\mathrm{C}$ ' in the inset is caused by the cryostat setup. 
Table 2

Structural parameters, fractional atomic coordinates and equivalent isotropic displacement parameters $\left(B_{\text {iso }}\right.$ in $\AA^{2}$ ) from the refinements of KNN-0.02BNT.

(a) SD refinements of $\mathrm{KNN}-0.02 \mathrm{BNT}$.

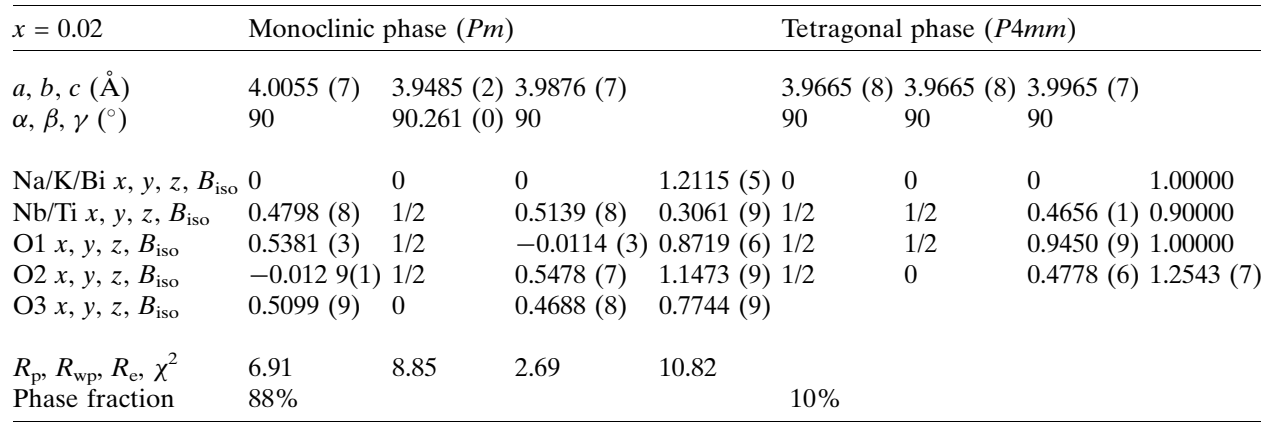

(b) Neutron refinement of $\mathrm{KNN}-0.02 \mathrm{BNT}$ at $4 \mathrm{~K}$.

\begin{tabular}{lllll}
\hline$x=0.02$ & \multicolumn{4}{l}{ Orthorhombic phase $(A m m 2)$ at $4 \mathrm{~K}$} \\
\hline$a, b, c(\AA)$ & $3.9503(4)$ & $5.6308(7)$ & $5.6583(8)$ & \\
$\alpha, \beta, \gamma\left({ }^{\circ}\right)$ & 90 & 90 & 90 & \\
$\mathrm{Na} / \mathrm{K} / \mathrm{Bi} x, y, z, B_{\text {iso }}$ & 0 & 0 & 0 & $1.2236(2)$ \\
$\mathrm{Nb} / \mathrm{Ti} x, y, z, B_{\text {iso }}$ & $1 / 2$ & 0 & $0.5067(4)$ & $0.6281(9)$ \\
$\mathrm{O} 1 x, y, z, B_{\text {iso }}$ & $1 / 2$ & $1 / 4$ & $0.2878(6)$ & $1.0146(3)$ \\
$\mathrm{O} 2 x, y, z, B_{\text {iso }}$ & 0 & 0 & $0.5419(6)$ & $0.7018(6)$ \\
$R_{\mathrm{p}}, R_{\mathrm{wp}}, R_{\mathrm{e}}, \chi^{2}$ & 8.34 & 9.42 & 1.90 & 24.53 \\
Phase fraction & $100 \%$ & & & \\
\hline
\end{tabular}

Phase fraction of impurity $\mathrm{K}_{6} \mathrm{Nb}_{10.88} \mathrm{O}_{30}$ is slightly below $2 \%$.

Table 3

Structural parameters, fractional atomic coordinates and equivalent isotropic displacement parameters $\left(B_{\text {iso }}\right.$ in $\left.\AA^{2}\right)$ from the SD refinements of the sample KNN-0.20BNT.

\begin{tabular}{|c|c|c|c|c|c|c|c|c|}
\hline$x=0.20$ & Tetragonal & phase $(P 4 b$ & $m)$ & & Cubic pha & se $(P m \overline{3} m)$ & & \\
\hline$a, b, c(\AA)$ & $5.5950(5)$ & $5.5950(5)$ & $3.9852(9)$ & & $3.9644(4)$ & $3.9644(4)$ & $3.9644(4)$ & \\
\hline$\alpha, \beta, \gamma\left(^{\circ}\right)$ & 90 & 90 & 90 & & 90 & 90 & 90 & \\
\hline $\mathrm{Na} / \mathrm{K} / \mathrm{Bi} x, y, z, B_{\text {iso }}$ & 0 & $1 / 2$ & $0.4594(4)$ & 1.00000 & 0 & 0 & 0 & $7.9631(7)$ \\
\hline $\mathrm{Nb} / \mathrm{Ti} x, y, z, B_{\text {iso }}$ & 0 & 0 & 0 & 0.90000 & $1 / 2$ & $1 / 2$ & $1 / 2$ & $0.5950(3)$ \\
\hline $\mathrm{O} 1 x, y, z, B_{\text {iso }}$ & 0 & 0 & $-0.0228(7)$ & 0.60000 & $1 / 2$ & $1 / 2$ & 0 & $1.0487(6)$ \\
\hline $\mathrm{O} 2 x, y, z, B_{\text {iso }}$ & $0.2476(4)$ & $0.2523(6)$ & $0.1001(6)$ & 0.26000 & & & & \\
\hline$R_{\mathrm{p}}, R_{\mathrm{wp}}, R_{\mathrm{e}}, \chi^{2}$ & 9.14 & 10.4 & 3.01 & 11.84 & & & & \\
\hline Phase fraction & $13 \%$ & & & & $85 \%$ & & & \\
\hline
\end{tabular}

Phase fraction of impurity $\mathrm{KTiNbO}_{5}$ is slightly above $2 \%$.

with the dielectric behavior and results from Raman spectroscopy (Liu et al., 2015), the structures within this region should be orthorhombic with Amm2 space group at room temperature. Furthermore, with the increase of BNT content, the orthorhombic-tetragonal phase transition shifts down to room temperature, resulting in an increase of the tetragonal phase fraction.

3.1.2. Rietveld analysis of powder diffraction data for $0.02 \leq x \leq 0.10$. For the samples with increasing BNT content from $x=0.02$ to $x=0.10$ the intensity of the Bragg reflection $002_{\mathrm{C}}$ decreases, while the intensity of $020_{\mathrm{C}}$ increases. This could point to the formation of a tetragonal phase. Therefore, two perovskite-type phases with $A m m 2$ and $P 4 m m$ symmetry were used for the calculation of a theoretical diffraction pattern, which was compared with the observed SD pattern ('Amm2 + $P 4 m m$ structure model'). However, the crystal structure of $\mathrm{KNN}$ is described by either a monoclinic $\left(a \simeq b \simeq c \simeq 4 \AA\right.$ А $\left.\beta \simeq 90.3^{\circ}\right)$ or an orthorhombic $\left[a \simeq c \simeq 4\left(2^{1 / 2}\right) \AA\right.$ and $b \simeq 4 \AA]$ unit cell (Tellier et al. 2009; Singh et al., 2001; Chu et al., 2003), leading to different indices $h k l_{\text {mono }}$ and $h k l_{\text {ortho, respectively. }}$ Since the width of the $002_{C}$ peak in $\mathrm{KNN}-0.02 \mathrm{BNT}$ is nearly twice that of the $200_{\mathrm{C}}$ peak (Fig. $3 a$ ), it was no longer possible to model the profile with an $A m m 2$ phase with anisotropic reflection broadening. Therefore, a monoclinic symmetry was considered, because the monoclinic perovskite phase with space group $\mathrm{Pm}$ is appropriate to describe the boundary between the orthorhombic phase with space group Amm2 and the tetragonal phase with space group $P 4 m m$, resulting from crystal lattice deformation and distortion (Kobayashi et al., 2012; Mgbemere et al., 2012). This alternative ' $P m+$ $P 4 \mathrm{~mm}$ structure model' was used for the fit to the experimental data as shown in Fig. 3(a). The structure model with space group $P m$, a subgroup of $A m m 2$ and $P 4 m m$, was derived from lattice parameters of the crystal lattice deformation and distortion caused by the BNT substitution. The refined structural parameters and goodness of fit obtained from the SD data are given in Table 2(a). The fit results are slightly better than that using the $A m m 2+P 4 m m$ model, with $R_{\mathrm{p}}=7.97 \%, R_{\mathrm{wp}}=9.90 \%$ and $R_{\mathrm{e}}=2.69 \%$. For the sample with $x=0.10$ the Bragg reflections $002_{\mathrm{C}}$ and $020_{\mathrm{C}}$ merge into one reflection. However, dielectric measurements indicate that the temperature of the dielectric maxima, $T_{\mathrm{m}}$, is about $500 \mathrm{~K}$, which suggests that the structure does not belong to cubic but to tetragonal symmetry at room temperature (Du et al., 2008). For the P4mm structure model alone no good agreement between the observed and calculated profiles was obtained for $x=0.10\left(R_{\mathrm{p}}=12.1 \%, R_{\mathrm{wp}}=\right.$ $13.7 \%$ and $R_{\mathrm{e}}=2.95 \%$ ) in comparison with the much better agreement for the $P m+P 4 m m$ structure model as shown in Fig. 3(b) $\left(R_{\mathrm{p}}=8.27 \%, R_{\mathrm{wp}}=9.52 \%\right.$ and $\left.R_{\mathrm{e}}=2.94 \%\right)$. The obtained phase ratios are $30 \% \mathrm{Pm}$ and $70 \% \mathrm{P} 4 \mathrm{~mm}$. In addi- 
Table 4

Space group, phase fraction and residuals obtained from Rietveld SD refinement for the sample $\mathrm{KNN}-0.50 \mathrm{BNT}$.

\begin{tabular}{llllll}
\hline Space group & Phase fraction $(\%)$ & $R_{\mathrm{p}}$ & $R_{\mathrm{wp}}$ & $R_{\mathrm{e}}$ & $\chi^{2}$ \\
\hline$R 3 c$ & 100 & 9.97 & 10.6 & 3.47 & 9.318 \\
$P m \overline{3} m$ & 100 & 11.0 & 10.5 & 3.49 & 9.089 \\
$P 4 m m$ & 100 & 12.1 & 12.1 & 3.53 & 11.64 \\
$R 3 c$ & 96 & 7.95 & 8.03 & 3.46 & 5.396 \\
$P 4 b m$ & 0.4 & & & & \\
$P 4 m m$ & 3.6 & & & & \\
$P m \overline{3} m$ & 40 & 6.70 & 6.59 & 3.37 & 3.817 \\
$P 4 b m$ & 60 & & & & \\
$P m \overline{3} m$ & 68 & 11.5 & 11.3 & 3.56 & 10.05 \\
$P 4 / m b m$ & 32 & & & & \\
$P m \overline{3} m$ & 94 & 15.0 & 17.7 & 3.53 & 25.09 \\
$P 4 m m$ & 6 & & & & \\
$R 3 m$ & 100 & 22.2 & 23.7 & 3.53 & 45.26 \\
$R 3 m$ & 7.4 & 12.0 & 12.7 & 3.46 & 13.41 \\
$P 4 b m$ & 92.6 & & & & \\
\hline
\end{tabular}

tion, a $P 4 m m+P 4 b m$ structure model with two tetragonal phases was checked $\left(R_{\mathrm{p}}=8.83 \%, R_{\mathrm{wp}}=9.62 \%\right.$ and $R_{\mathrm{e}}=$ $2.94 \%$ ) but failed against the $P m+P 4 m m$ model. Therefore, the most convincing structure model in the region $0.02 \leq x \leq$ 0.10 should exhibit a coexistence of monoclinic and tetragonal symmetry.

Dielectric measurements indicate a phase transition from orthorhombic to rhombohedral at a transition temperature of $\sim 140 \mathrm{~K}$ for pure $\mathrm{KNN}$, which shifts to lower temperatures with the addition of BNT (Du et al., 2008). Therefore, a different low-temperature structure is also expected for (1 $x) \mathrm{KNN}-x \mathrm{BNT}$. Neutron diffraction was measured at $4 \mathrm{~K}$ for the sample with $x=0.02$ as shown in Fig. 3(c). This is a singlephase perovskite structure with $A m m 2$ space group. The reflection marked with an arrow and labeled as $\mathrm{C}$ (at $2 \theta=$ $38.1^{\circ}$ ) in the diffraction pattern of the orthorhombic phase is attributed to the cryostat material [see the inset of Fig. 3(c)]. The detailed structural parameters and goodness of fit obtained from neutron data are given in Table $2(b)$. The

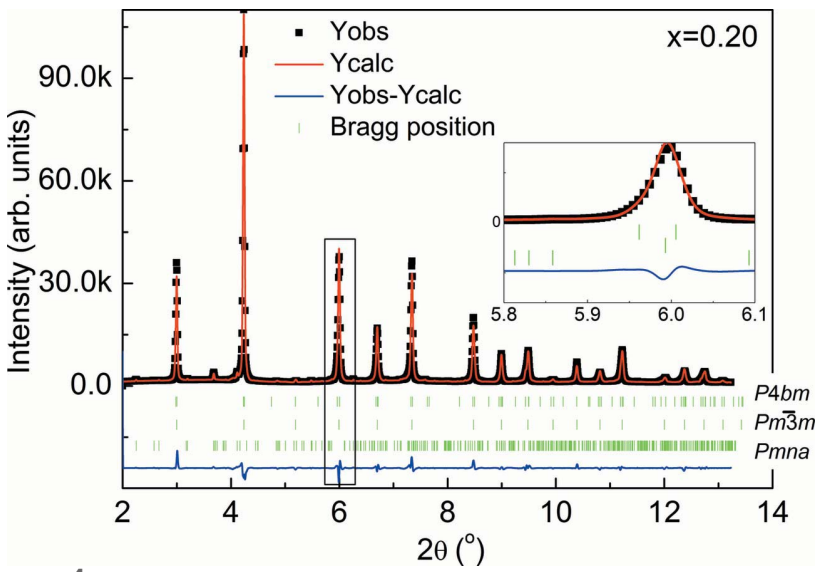

Figure 4

Rietveld refinements based on synchrotron diffraction data of KNN$0.20 \mathrm{BNT}$. The inset shows the regions around the $200_{\mathrm{C}}$ reflection with contributions from the $P 4 b m$ phase and the $P m \overline{3} m$ phase. results suggest that the orthorhombic phase of KNN is stabilized at low temperatures by BNT.

3.1.3. Rietveld analysis of powder diffraction data for $0.20 \leq x \leq 0.50$. The structure analysis for this region is challenging, because all structures appear pseudocubic in the SD patterns. Nevertheless, the Rietveld fits based on either a $P m \overline{3} m$ or a $P m+P 4 m m$ structure model do not converge with low $R$ values. However, the fit is satisfactory for a $\operatorname{Pm} \overline{3} m+$ $P 4 b m$ structure model as shown in Fig. 4 and Table 3 . The fraction of about $13 \% \mathrm{P} 4 \mathrm{bm}$ indicates an octahedral tilting in the sample with $x=0.20$, which is very different from the structure in the sample with $x=0.10$. In order to determine the octahedral tilting in this region more reliably, neutron diffraction was performed on a sample with $x=0.30$ at room temperature and at $4 \mathrm{~K}$. The $P 4 b m$ phase fraction in this sample is $7.4 \%$ at room temperature and slightly increases with decreasing temperature. Fig. 5(a) shows the neutron diffraction pattern of the sample with $x=0.30$ at $4 \mathrm{~K}$. The $\frac{1}{2} 311$ and $\frac{1}{2} 531$ superlattice reflections are not detected, but weak $\frac{1}{2} 530$ and $\frac{1}{2} 532$ superlattice reflections appear, as shown in the inset of Fig. 5(a). The $\frac{1}{2}$ ooo and $\frac{1}{2}$ ooe superlattice reflections

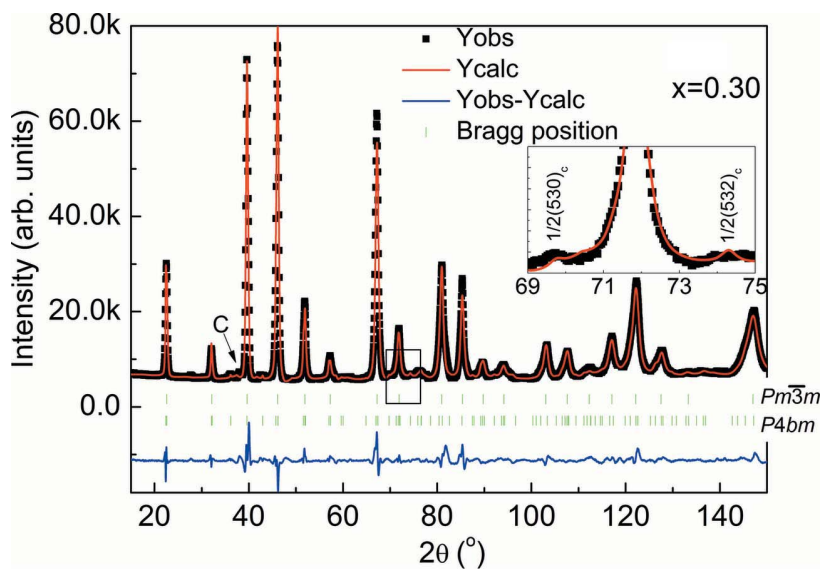

(a)

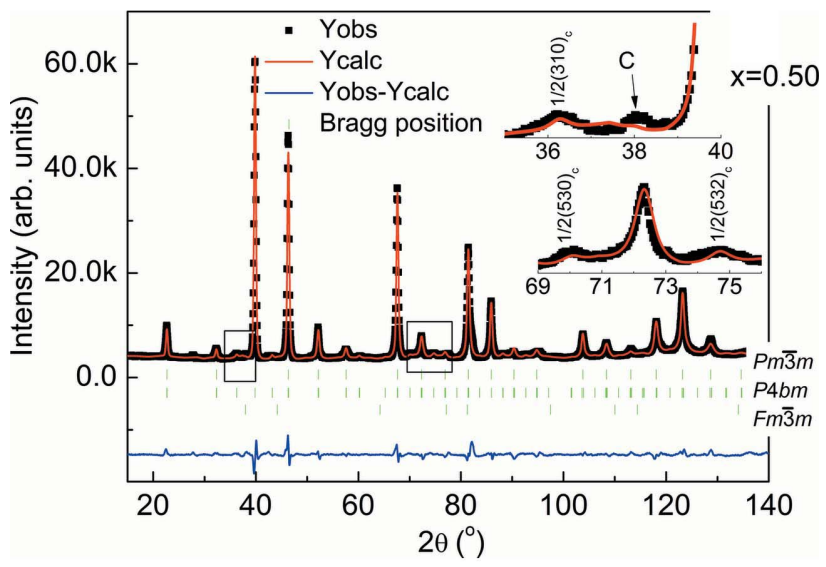

(b)

Figure 5

(a) Rietveld refinements based on neutron diffraction data at $4 \mathrm{~K}$ for $\mathrm{KNN}-0.30 \mathrm{BNT}$. Weak tetragonal superlattice reflections ooe are visible in the inset, but no rhombohedral superlattice reflections. (b) The same, but for KNN-0.50BNT. The inset shows the attribution of the characteristic superlattice reflections ooe appearing in the $\mathrm{P} 4 \mathrm{bm}$ phase. The reflection at $2 \theta=38.1^{\circ}$ marked with ' $\mathrm{C}$ ' is due to the cryostat material. 
Table 5

Structural parameters, fractional atomic coordinates and equivalent isotropic displacement parameters $\left(B_{\text {iso }}\right.$ in $\left.\AA^{2}\right)$ from the neutron refinements of the sample of KNN-0.50BNT at $4 \mathrm{~K}$.

\begin{tabular}{|c|c|c|c|c|c|c|c|c|}
\hline$x=0.50$ at $4 \mathrm{~K}$ & Tetragonal & phase $(P 4$ & $b m)$ & & Cubic pha & se $(P m \overline{3} m)$ & & \\
\hline$a, b, c(\AA)$ & $5.5615(2)$ & $5.5615(2)$ & 3.9397 (4) & & $3.9359(3)$ & $3.9359(3)$ & 3.9359 (3) & \\
\hline$\alpha, \beta, \gamma\left({ }^{\circ}\right)$ & 90 & $90.261(2)$ & 90 & & 90 & 90 & 90 & \\
\hline $\mathrm{Na} / \mathrm{K} / \mathrm{Bi} x, y, z, B_{\text {iso }}$ & 0 & $1 / 2$ & $0.6117(5)$ & $6.4416(2)$ & 0 & 0 & 0 & $3.5721(8)$ \\
\hline $\mathrm{Nb} / \mathrm{Ti} x, y, z, B_{\text {iso }}$ & 0 & 0 & 0 & $0.0336(4)$ & $1 / 2$ & $1 / 2$ & $1 / 2$ & $0.6264(3)$ \\
\hline $\mathrm{O} 1 x, y, z, B_{\text {iso }}$ & 0 & 0 & $0.4698(7)$ & $0.0708(7)$ & $1 / 2$ & $1 / 2$ & 0 & $3.3468(3)$ \\
\hline $\mathrm{O} 2 x, y, z, B_{\text {iso }}$ & $0.2676(9)$ & $0.2323(1)$ & $-0.0493(5)$ & $0.2087(6)$ & & & & \\
\hline$R_{\mathrm{p}}, R_{\mathrm{wp}}, R_{\mathrm{e}}, \chi^{2}$ & 13.6 & 13.1 & 3.54 & 13.81 & & & & \\
\hline Phase fraction & $45 \%$ & & & & $55 \%$ & & & \\
\hline
\end{tabular}

Table 6

Structural parameters, fractional atomic coordinates and equivalent isotropic displacement parameters $\left(B_{\text {iso }}\right.$ in $\AA^{2}$ ) from the neutron refinements of the sample of KNN-0.90BNT at $300 \mathrm{~K}$.

\begin{tabular}{|c|c|c|c|c|c|c|c|}
\hline \multirow{2}{*}{$\begin{array}{l}x=0.90 \text { at } 300 \mathrm{~K} \\
a, b, c(\AA)\end{array}$} & \multicolumn{4}{|c|}{ Tetragonal phase $(P 4 b m)$} & \multicolumn{3}{|c|}{ Rhombohedral phase $(R 3 c)$} \\
\hline & $5.51299(1)$ & 5.5117 & $3.8974(2)$ & & 5.5120 (4) $5.5120(4)$ & 13.514 & \\
\hline$\alpha, \beta, \gamma\left({ }^{\circ}\right)$ & 90 & 90 & 90 & & $90 \quad 90$ & 120 & \\
\hline $\mathrm{Na} / \mathrm{K} / \mathrm{Bi} x, y, z, B_{\text {iso }}$ & 0 & $1 / 2$ & $0.5777(6)$ & $3.7171(5)$ & 0 & $0.2573(4)$ & $4.3298(2)$ \\
\hline $\mathrm{Nb} / \mathrm{Ti} x, y, z, B_{\text {iso }}$ & 0 & 0 & 0 & 0.9250 & 0 & $-0.0133(9)$ & $0.9359(8)$ \\
\hline $\mathrm{O} 1 x, y, z B_{\text {iso }}$ & 0 & 0 & $0.5396(6)$ & $1.9364(9)$ & 0.1480 & $0.0833(3)$ & $3.7436(2)$ \\
\hline $\mathrm{O} 2 x, y, z, B_{\text {iso }}$ & $0.2745(7)$ & $0.2254(3)$ & $0.0670(4)$ & $3.2238(1)$ & & & \\
\hline$R_{\mathrm{p}}, R_{\mathrm{wp}}, R_{\mathrm{e}}, \chi^{2}$ & 19.8 & 12.7 & 5.21 & 5.98 & & & \\
\hline Phase fraction & $22 \%$ & & & & $78 \%$ & & \\
\hline
\end{tabular}

show a weak superlattice reflection $\frac{1}{2} 311$ near the Bragg reflection $111_{\mathrm{C}}$, which becomes stronger with an increase of the BNT content. The space group $R 3 c$ was initially chosen, because it has been reported for compositions of the $\mathrm{Bi}_{1 / 2} \mathrm{Na}_{1 / 2} \mathrm{TiO}_{3}-\mathrm{BaTiO}_{3}-\mathrm{K}_{0.5} \mathrm{Na}_{0.5} \mathrm{NbO}_{3}$ system from both TEM and SD studies (Schmitt \& Kleebe, 2010; Schmitt \& Kleebe, 2010). For the sample with $x=0.90$, a single $R 3 c$ model yielded relatively high $R$ values: $R_{\mathrm{p}}=9.18 \%, R_{\mathrm{wp}}=8.32 \%$ and $R_{\mathrm{e}}=3.29 \%$. Therefore, a mixed $R 3 c+P 4 b m$ model was used and gave a reasonably good quality of fit $\left(R_{\mathrm{p}}=6.37 \%\right)$, shown in Fig. 6(a). Since the superlattice reflections of $\mathrm{P} 4 \mathrm{bm}$ are not visible in the SD patterns, neutron diffraction on KNN-90BNT was carried out. The pattern exhibits $\frac{1}{2}$ ooo and $\frac{1}{2}$ ooe superlattice reflections, which belong to the $R 3 c$ and $\mathrm{P} 4 \mathrm{bm}$ space groups, respectively (Woodward \& Reaney, 2005; Glazer, 1975). The mixed $R 3 c+$

indicate the presence of $a^{-} a^{-} a^{-}$and $a^{0} a^{0} a^{+}$octahedral tiltings (Woodward \& Reaney, 2005; Kishida et al., 2009), respectively. The appearance of $\frac{1}{2}$ ooe superlattice reflections implies the existence of a tetragonal phase. Therefore, the $P 4 b m+P m \overline{3} m$ model is reasonable, and observed and calculated profiles agree well: $R_{\mathrm{p}}=10.8 \%, R_{\mathrm{wp}}=11.6 \%$ and $R_{\mathrm{e}}=2.25 \%$.

For the analysis of the high-resolution SD pattern from the sample with $x=0.50$, several space groups were considered, including $R 3 c, R 3 m, P 4 / m b m, P 4 b m, P 4 m m, P m \overline{3} m$ and multiphase models with combinations of these (see Table 4$)$. The best fit was obtained for a multiphase mixture of $P 4 b m+$ $P m \overline{3} m$. Similar results were obtained from room-temperature neutron diffraction patterns.

In order to detect the phase transition behavior from pseudocubic to tetragonal, low-temperature neutron diffraction experiments were performed. Fig. $5(b)$ shows the neutron diffraction pattern of the sample with $x=0.50$ at $4 \mathrm{~K}$. The Bragg reflections 111 and 200 are not split, so no obvious phase transition is indicated. However, the reflection at $2 \theta=$ $36.8^{\circ}$ becomes more clear and an additional reflection appears at $2 \theta=38.1^{\circ}$. The reflection at $2 \theta=36.8^{\circ}$ should correspond to $\frac{1}{2} 310$ of the $P 4 b m$ phase, while the intensities at $2 \theta=38.1^{\circ}$ appear in all low-temperature patterns independent of composition and are again assigned to some scattering from the cryostat ('C'). The refined parameters for the $P 4 b m+$ $P m \overline{3} m$ structure model are given in Table 5, while observed and calculated profiles are shown in Fig. 5(b).

3.1.4. Rietveld analysis of powder diffraction data for $0.90 \leqq x \leqq 1.00$. The SD patterns of this composition range
$P 4 b m$ structure model was refined on the basis of neutron data as shown in Fig. 6(b). The lattice parameters and goodness of fit are listed in Table 6. The isotropic atomic displacement parameter $\left(B_{\text {iso }}\right)$ of the $A$-site cations was found to increase considerably as the composition approaches the BNT-rich end. The same trend is also observed for the $B_{\text {iso }}$ values of the oxygen atoms. Contrary to the $B_{\text {iso }}$ values of the $A$-site cations and oxygen, the $B_{\text {iso }}$ values of Ti/Nb are not affected significantly by changes in composition $x$. The increase in the $B_{\text {iso }}$ values of $\mathrm{Na} / \mathrm{K} / \mathrm{Bi}$ with increasing $\mathrm{BNT}$ fraction suggests an increase in static disorder on the $A$ site of the structure (Ranjan et al., 2010). One of the reasons for the large static disorder could be related to the presence of three different types of ions $\left(\mathrm{Na}^{+}, \mathrm{Bi}^{3+}\right.$ and $\left.\mathrm{K}^{+}\right)$with different ionic radii occupying the $A$ site of the $A B \mathrm{O}_{3}$ structure.

The neutron diffraction pattern of the sample at $4 \mathrm{~K}$ is shown in Fig. 6(c). The $\frac{1}{2} 531$ superlattice reflection and both $\frac{1}{2} 530$ and $\frac{1}{2} 532$ superlattice reflections are clearly visible in the pattern. Furthermore, the Bragg reflections 111 and 200 in the neutron pattern are not split, which excludes a complete phase transition from rhombohedral to tetragonal. Therefore, a mixed $R 3 c+P 4 b m$ model was used and refined: $R_{\mathrm{p}}=19.4 \%$, $R_{\text {wp }}=13.7 \%$ and $R_{\mathrm{e}}=3.67 \%$. The phase fraction of $R 3 c$ and $P 4 \mathrm{bm}$ is 85 and $15 \%$, respectively.

\subsection{Transmission electron microscopy}

In order to reveal subtle deviations from a cubic structure of the samples with $x=0.50$ and $x=0.90$, a TEM-selected area 
electron diffraction (SAED) study of the systematic presence or absence of superstructure reflections in two different zone axes was performed. To simplify matters, zone axes are indexed as $[h k l]_{\mathrm{C}}$, corresponding to the primitive cubic aris-

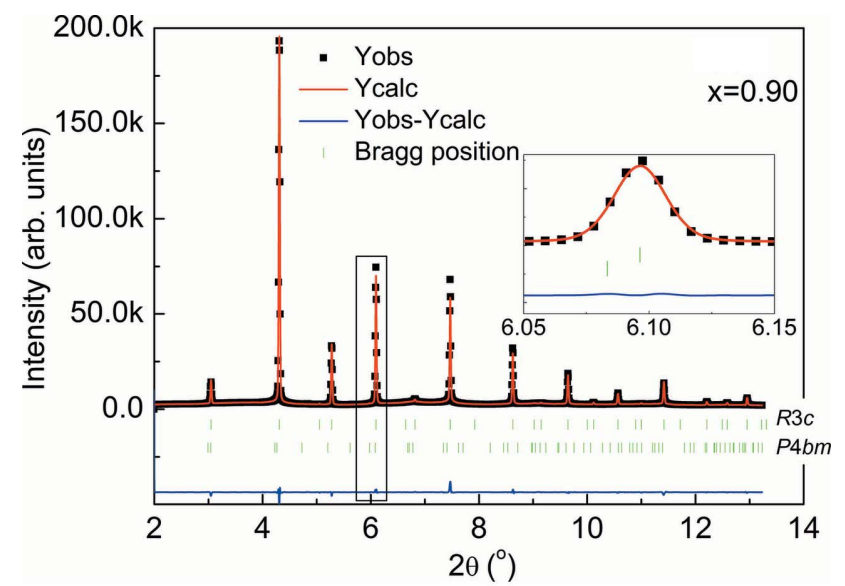

(a)

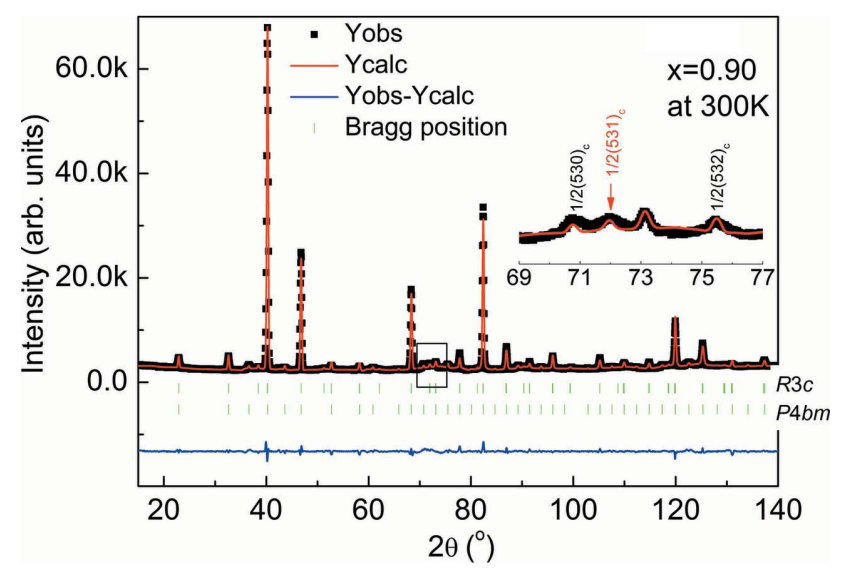

(b)

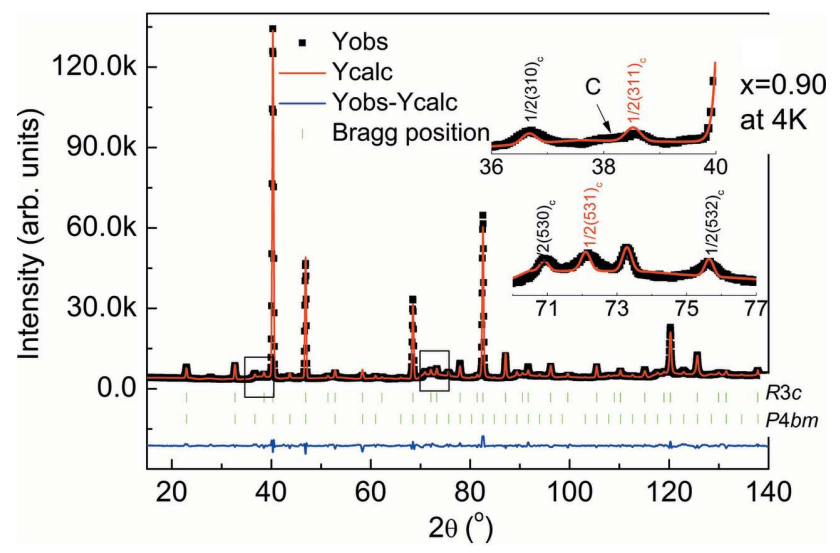

(c)

Figure 6

(a) Rietveld refinements based on synchrotron diffraction data of KNN$0.90 \mathrm{BNT}$. The inset shows the region around the $200_{\mathrm{C}}$ reflection with contributions from the tetragonal $P 4 b m$ phase and rhombohedral $R 3 c$ phase. $(b),(c)$ The same, but for neutron diffraction data $(b)$ at room temperature and $(c)$ at $4 \mathrm{~K}$. Both rhombohedral and tetragonal superstructure reflections ooe and $o o o$, respectively, are visible in the insets. The reflection at $2 \theta=38.1^{\circ}$ marked with ' $\mathrm{C}$ ' is due to the cryostat material. totype structure. In Fig. 7, SAED patterns from two samples are shown, with $x=0.50$ (left column) and $x=0.90$ (right column). The $\frac{1}{2}$ ooo or/and $\frac{1}{2}$ ooe superstructure reflections are due to $a^{-} a^{-} a^{-}$and $a^{0} a^{0} c^{+}$octahedral tiltings and are consistent with the synchrotron and neutron diffraction data. In the $[001]_{\mathrm{C}}$ zone, the $\frac{1}{2}$ ooe superstructure reflections from the tetragonal phase are visible. The $\frac{1}{2}$ ooo superstructure reflections result from the rhombohedral phase visible in the $[011]_{C}$ zone. Both types of superstructure reflections are detected in the $[013]_{\mathrm{C}}$ zone.

Figs. 7(a) and 7(b) show the SAED patterns of the $[001]_{\mathrm{C}}$ zone axis. Reflections appear as double spots for the sample with $x=0.50$, but these virtual splittings are overlaps from diffraction patterns of two slightly differently oriented domains. Both samples show $\frac{1}{2}$ ooe superlattice reflections, revealing a proportion of the tetragonal phase, consistent with a previous study of the BNT-BT-KNN system (Schmitt et al., 2010; Schmitt \& Kleebe, 2010; Kling et al., 2010). In Figs. 7(c) and $7(d)$, SAED patterns along $[110]_{\mathrm{C}}$ are depicted. The $\frac{1}{2}$ ooo superstructure reflections in Fig. $7(d)$ are strongly excited, whereas in Fig. 7(c) they are invisible. This leads to the conclusion that the rhombohedral phase fraction is not present in the sample with $x=0.50$ but present in the sample with $x=0.90$. These results again agree very well with the observations from synchrotron and neutron diffraction experiments.
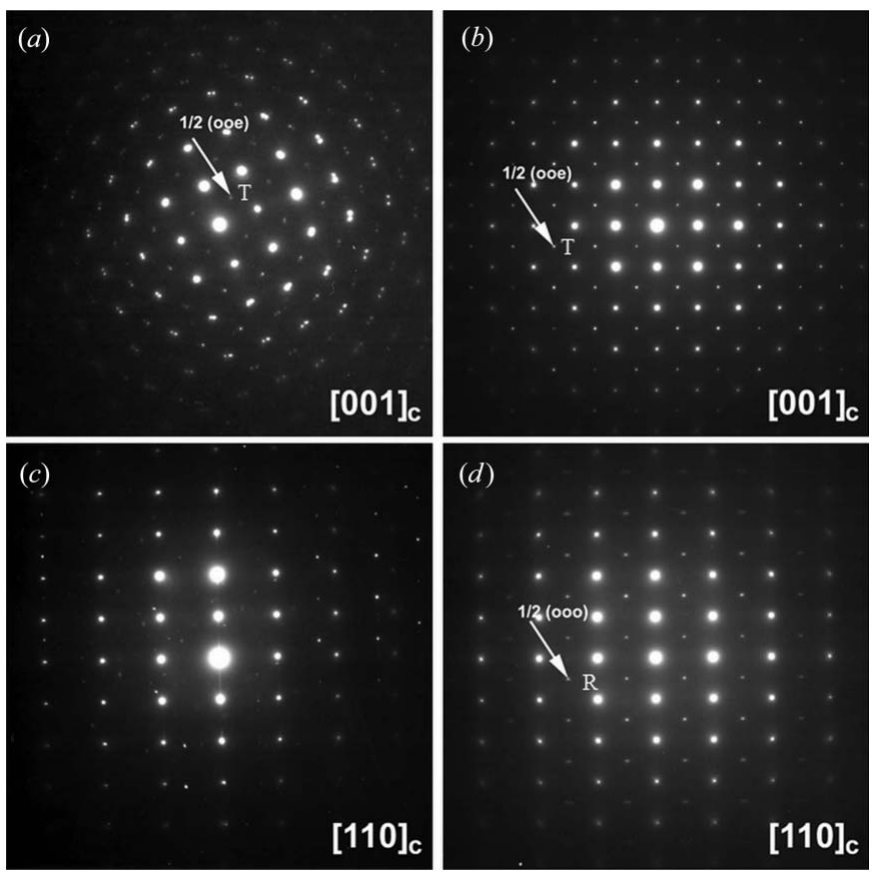

Figure 7

SAED patterns of the $[001]_{\mathrm{c}}$ and $[110]_{\mathrm{c}}$ zone axes. Arrows indicate the $\frac{1}{2}$ ooo and $\frac{1}{2}$ ooe superstructure reflections, where $R$ means rhombohedral and $T$ tetragonal. The columns show the zone axis diffraction patterns for the sample with $x=0.50$ (left) and for $x=0.90$ (right). (a), (b) Clearly visible intensity from $\frac{1}{2}$ ooe superlattice reflections, due to a fraction of the tetragonal phase. (c) No $\frac{1}{2}$ ooo superlattice reflections, owing to the absence of the rhombohedral phase. (d) Clearly visible intensity from $\frac{1}{2}$ ooo superstructure reflections, signifying a fraction of the rhombohedral phase. 
In a previous paper (Schmitt et al., 2010; Schmitt \& Kleebe, 2010), the ternary system containing BNT, which is solely rhombohedral, BT with a tetragonal structure and orthorhombic KNN has been studied. Considering this mixture with four different $A$-site cations and two different $B$-site cations, a local accumulation of specific cations could lead to a stabilization of one of the three different structures. Likewise, Dorcet \& Trolliard (2008) reported a stabilization of the tetragonal structure, induced by the presence of $\mathrm{K}^{+}$and $\mathrm{Ba}^{2+}$ ions. Comparing the samples studied, the one with $x=0.50$ has a higher proportion of $\mathrm{Na}^{+}$and $\mathrm{K}^{+}$and shows a coexistence of the pseudocubic and tetragonal phases without the rhombohedral phase, whereas with the increase of $\mathrm{Bi}^{3+}$ content, as for example in the sample with $x=0.90$, the rhombohedral phase is predominant. This suggests that the $A$-site $\mathrm{Bi}^{3+}$ ion with a lone $6 s^{2}$ electron pair plays a key role in the structure of the KNN-BNT system (Schütz et al., 2012).

Electron diffraction studies of BNT exhibit localized inphase $(+)$ octahedral tilts in the otherwise average $a^{-} a^{-} a^{-}$/ $a^{-} a^{-} c^{-}$tilt present in this system (Dorcet et al., 2008; Trolliard $\&$ Dorcet, 2008). Two alternative views are possible: (i) BNT is a two-phase mixture with the major phase being rhombohedral ( $R 3 c$, tilt system $\left.a^{-} a^{-} a^{-}\right)$, along with small tetragonal platelets $\left(P 4 b m\right.$, tilt system $\left.a^{0} a^{0} c^{+}\right)$, at room temperature (Kreisel et al., 2003, 2004); or (ii) nanoscale domains exist with very short range $a^{-} a^{-} c^{+}$tilts (1-3 nm), interspersed in between the relatively long range $a^{-} a^{-} c^{-} / a^{-} a^{-} a^{-}$tilts (10$40 \mathrm{~nm}$ ) revealed by electron diffraction (Levin \& Reaney, 2012). This short range alteration in the tilt sequence occurs across pseudocubic $100_{\mathrm{C}}$ twin domain boundaries. For the latter model, such an assemblage of octahedral disorder would lead to an average out-of-phase tilting along any octahedral chain, and yield a pseudo-rhombohedral/monoclinic structure (Rao et al., 2013). The consideration of complex multiphase models has improved the description of the underlying structures, but some features are still not fully explained. They are probably caused by local disorder, which has not yet been sufficiently addressed. Such a feature is the large $A$-site displacement parameter in BNT-rich phases. Large $A$-site displacement parameter values $\left(\sim 0.054 \AA^{2}\right)$ have also been reported in the related ternary system $\mathrm{Bi}_{1 / 2} \mathrm{Na}_{1 / 2} \mathrm{TiO}_{3}-$ $\mathrm{BaTiO}_{3}-\mathrm{K}_{0.5} \mathrm{Na}_{0.5} \mathrm{NbO}_{3}$ (Schmitt et al., 2010; Schmitt \& Kleebe, 2010). Typical values for perovskite ferroelectric or relaxor materials with only one $A$-site cation (e.g. $\mathrm{BaTiO}_{3}$, $\mathrm{PbMg}_{1 / 3} \mathrm{Nb}_{2 / 3} \mathrm{O}_{3}$ and $\mathrm{PbZr}_{0.52} \mathrm{Ti}_{0.48} \mathrm{O}_{3}$ ) range from $\sim 0.001$ to $\sim 0.01 \AA^{2}$ (Kwei et al., 1993; Bonneau et al., 1991; Noheda et $a l ., 2000)$, less than one-fifth of the obtained values for (1 $x) \mathrm{KNN}-x \mathrm{BNT}$ materials. These large displacement parameters may indicate a pronounced thermal motion of the atoms on this site. However, the fact that the ionic radii of the $A$-site cations are large does not support this and a static disorder, reflected by a distribution of lattice parameters or lattice distortions due to the three different $A$-site cations, seems more probable (Usher et al., 2012).

Liu et al. (2015) reported that the temperature corresponding to the maximum value $\left(\varepsilon_{\mathrm{m}}^{\prime}\right)$ of the real part of the dielectric permittivity, $T_{\mathrm{m}}$, decreases with the increase of BNT.
The minimum of $T_{\mathrm{m}}$ is $\sim 350 \mathrm{~K}$ for the sample of $x=0.50$. Moreover, $\varepsilon_{\mathrm{m}}$ for $x>0.50$ shifts toward higher temperatures with increasing $x$. The change in dielectric behavior follows the sequence normal ferroelectric, diffuse phase transition, reentrant-like relaxor, relaxor/glasslike, BNT-like relaxor with the increase of BNT. Raman spectroscopy shows that the maximum of mode softening/hardening at $x=0.06,0.50$ and 0.90 indicates phase boundaries or changes of local structure at these compositions. Fig. 8 combines and summarizes all experimental results from this work and related studies of BNT-based solid solutions (Ma et al., 2010; Ma \& Tan, 2010, 2011; Hiruma et al., 2008) to derive a phase diagram for the quasi-binary $(1-x) \mathrm{KNN}-x \mathrm{BNT}$ system. On the KNN-rich side of the phase diagram, the structure undergoes a transition from single $A m m 2$ to a mixture of $P 4 m m+P m$ at room temperature with increasing BNT content. Octahedral tilts appear as described in the $\mathrm{P} 4 \mathrm{bm}$ structure model for increasing concentrations of BNT from $\sim 14 \%$ at room temperature. A very broad phase mixture is present in the range $0.14<x \leq 0.87$, accompanied by ion disorder on both $A$ and $B$ sites. In the range $0.87<x \leq 1.00$ the $P 4 b m+R 3 c$ phase mixtures change into a single $R 3 c$ phase at $x \simeq 0.96$ with the increase of BNT content. This is in agreement with the AFEFE transition revealed by the electromechanical behavior and reported as an MPB (Kounga et al., 2008).

At room temperature, four distinct phase boundaries can be defined in the phase diagram. The PPB behavior and tilt system induced by BNT lead to a complex phase evolution, although the structural change near phase coexistence regions is small compared to that in PZT. It is not necessarily reasonable to refer to these regions $(0.02<x<0.14$ and $0.87<$ $x<0.96)$ as MPBs, as their character differs from that of PZT. In terms of previous studies (Jaffe et al., 1971; Noheda et al., 1999), an MPB should possess the following characteristics (Dai et al., 2007): (i) An unlimited solid solution is formed from two components with almost the same type of structure, and the characteristic of an MPB region is compositional homogeneity. (ii) It is difficult to define the exact composition location of an MPB although the MPB was initially defined as

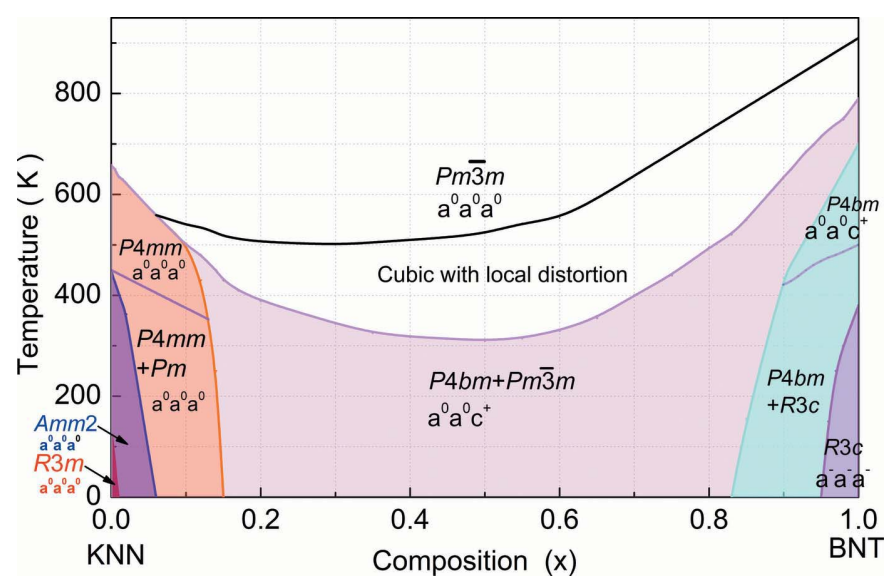

Figure 8

Phase diagram for the KNN-BNT system, including the assignment of space groups and oxygen tilting schemes for each phase. 
a boundary between two phases. (iii) The crystal structure of an MPB region is not dependent on temperature in a narrow composition range, which is the key difference from the PPB. Characteristics (i) and (ii) are applicable to the $(1-x) \mathrm{KNN}-$ $x$ BNT system. However, for the third point, the two regions $0.02<x<0.14$ and $0.87<x<0.96$ in the $(1-x) \mathrm{KNN}-x \mathrm{BNT}$ system need to go through a tetragonal phase to reach cubic symmetry with the increase of temperature. The phase structure (or phase fraction) is very sensitive to the temperature, although good piezoelectric properties can be obtained near room temperature (Zuo et al., 2007; Kounga et al., 2008).

The MPB often includes a narrow region with two-phase coexistence or represents a low-symmetry transitional phase. An intermediate monoclinic phase was found in a narrow region at the MPB of PZT (Guo et al., 2000). The monoclinic phase allows a continuous rotation of the polarization direction from $R$ ([111]) to $T$ ([001]). The region $x \simeq 0.96$ in the $(1-x) \mathrm{KNN}-x \mathrm{BNT}$ system should be investigated further to reveal the large strain contributed by the phase coexistence of $R$ and $T$ phases. However, the identification of an intermediate phase by diffraction is more difficult for $(1-x) \mathrm{KNN}-x \mathrm{BNT}$ than for PZT, owing to the larger disorder caused by the coexistence of ions with different charges, $\mathrm{Na}^{+}, \mathrm{K}^{+}$and $\mathrm{Bi}^{3+}$ in the $A$ sublattice and $\mathrm{Ti}^{4+}$ and $\mathrm{Nb}^{5+}$ in the $B$ sublattice.

The monoclinic phase is not an intermediate phase between orthorhombic and tetragonal phases, although it is present in the range $0.02<x<0.14$, but is better described as a PPB instead of an MPB. Since space group Pm belongs to the subgroups of $A m m 2$ and $P 4 m m$, it allows a continuous rotation of the polarization direction from orthorhombic ([110]) to tetragonal ([100]) (Mgbemere et al., 2012). Therefore, the role of the monoclinic phase in the $(1-x) \mathrm{KNN}-x \mathrm{BNT}$ system is similar to that in PZT, except that the specific directions of polarization are different.

\section{Conclusions}

In this study, a combination of synchrotron, neutron and electron diffraction has provided a more detailed insight into the material structure and phase transitions of the quasibinary $(1-x) \mathrm{KNN}-x \mathrm{BNT}$ solid solution system. A phase diagram is proposed, including space groups and structure models for all involved phases. This review in combination with detailed diffraction studies resolves certain ambiguities in the literature. The structures of the orthorhombic, monoclinic, tetragonal and rhombohedral phases and the phase coexistence of monoclinic + tetragonal in the range $0.02<x \leq 0.14$, tetragonal + pseudocubic for $0.14<x \leq 0.87$ and tetragonal + rhombohedral for $0.87<x \leq 0.96$ have been confirmed and subtle changes in the underlying structures were observed. The oxygen octahedral tilt systems $a^{0} a^{0} a^{+}$and $a^{-} a^{-} a^{-}$ successively appear with the increase of BNT content. Four distinct phase boundaries can be found in the phase diagram at room temperature; however, the phase-coexistence regions $(0.02<x<0.14$ and $0.87<x<0.96)$ between phase boundaries should be assigned as a PPB rather than an MPB. Understanding the structure evolution of lead-free piezoceramics elucidates the composition dependence of their properties in terms of the underlying structures and allows tailoring of the desired materials for enhanced applications.

\section{Acknowledgements}

This work was financially supported by a fellowship from a program for visiting scientists at the Helmholtz centre KIT. It also benefitted from beamtime allocation at P02.1 (PETRA III, DESY) as well as at SPODI (FRM II, MLZ), with access supported by the Bundesministerium für Bildung und Forschung under grant No. 05K13VK1 and by the Australian Research Council (ARC) under grant No. DE150100750. The Natural Science Foundation of China (grant Nos. 11264010, 51002036 and 11564010) and the Natural Science Foundation of Guangxi (grant Nos. GA139008 and FA139003) are also acknowledged. Helpful discussions with Professor Dhananjai Pandey and Dr Ravindra Singh Solanki are gratefully acknowledged.

\section{References}

Aksel, E., Forrester, J. S., Jones, J. L., Thomas, P. A., Page, K. \& Suchomel, M. R. (2011). Appl. Phys. Lett. 98, 152901.

Bonneau, P., Garnier, P., Calvarin, G., Husson, E., Gavarri, J. R., Hewat, A. W. \& Morell, A. (1991). J. Solid State Chem. 91, 350-361. Chu, S. Y., Water, W., Juang, Y. D. \& Liaw, J. T. (2003). Ferroelectrics, 287, 23-33.

Dai, Y., Zhang, X. \& Zhou, G. (2007). Appl. Phys. Lett. 90, 262903.

Dorcet, V. \& Trolliard, G. (2008). Acta Mater. 56, 1753-1761.

Dorcet, V., Trolliard, G. \& Boullay, P. (2008). Chem. Mater. 20, 50615073.

Du, H., Zhou, W., Luo, F., Zhu, D., Qu, S., Li, Y. \& Pei, Z. (2008). J. Phys. D Appl. Phys. 41, 085416.

Fu, J., Zuo, R. \& Liu, Y. (2010). J. Alloys Compd. 493, 197-201.

Glazer, A. M. (1975). Acta Cryst. A31, 756-762.

Gorfman, S., Glazer, A. M., Noguchi, Y., Miyayama, M., Luo, H. \& Thomas, P. A. (2012). J. Appl. Cryst. 45, 444-452.

Gorfman, S. \& Thomas, P. A. (2010). J. Appl. Cryst. 43, 1409-1414.

Guo, R., Cross, L. E., Park, S.-E., Noheda, B., Cox, D. E. \& Shirane, G. (2000). Phys. Rev. Lett. 84, 5423-5426.

Herklotz, M., Scheiba, F., Hinterstein, M., Nikolowski, K., Knapp, M., Dippel, A.-C., Giebeler, L., Eckert, J. \& Ehrenberg, H. (2013). J. Appl. Cryst. 46, 1117-1127.

Hiruma, Y., Nagata, H. \& Takenaka, T. (2008). J. Appl. Phys. 104, 124106.

Hoelzel, M., Senyshyn, A., Juenke, N., Boysen, H., Schmahl, W. \& Fuess, H. (2012). Nucl. Instrum. Methods Phys. Res. Sect. A, 667, 32-37.

Jaffe, B., Cook, W. R. \& Jaffe, H. (1971). Piezoelectric Ceramics. New York: Academic Press.

Jones, G. O. \& Thomas, P. A. (2000). Acta Cryst. B56, 426-430.

Jones, G. O. \& Thomas, P. A. (2002). Acta Cryst. B58, 168-178.

Kishida, K., Goto, K. \& Inui, H. (2009). Acta Cryst. B65, 405-415.

Kling, J., Tan, X., Jo, W., Kleebe, H.-J., Fuess, H. \& Rödel, J. (2010). J. Am. Ceram. Soc. 93, 2452-2455.

Kobayashi, K., Hatano, K., Mizuno, Y. \& Randall, C. A. (2012). Appl. Phys. Expr. 5, 031501.

Kounga, A. B., Zhang, S., Jo, W., Granzow, T. \& Rödel, J. (2008). Appl. Phys. Lett. 92, 222902.

Kreisel, J., Bouvier, P., Dkhil, B., Chaabane, B., Glazer, A. M., Thomas, P. A. \& Welberry, T. R. (2004). Ferroelectrics, 302, 293 298. 
Kreisel, J., Bouvier, P., Dkhil, B., Thomas, P. A., Glazer, A. M., Welberry, T. R., Chaabane, B. \& Mezouar, M. (2003). Phys. Rev. B, 68, 014113 .

Kwei, G. H., Lawson, A. C., Billinge, S. J. L. \& Cheong, S. W. (1993). J. Phys. Chem. 97, 2368-2377.

Levin, I. \& Reaney, I. M. (2012). Adv. Funct. Mater. 22, 3445-3452.

Liu, L., Huang, Y., Li, Y., Fang, L., Dammak, H., Fan, H. \& Thi, M. (2012). Mater. Lett. 68, 300-302.

Liu, L., Knapp, M., Ehrenberg, H., Fang, L., Schmitt, L., Fuess, H., Hoelzel, M., Dammak, H., Thi, M. \& Hinterstein, M. (2015). arXiv: 1510.05364 .

Liu, L., Wu, M., Huang, Y., Fang, L., Fan, H., Dammak, H. \& Thi, M. (2011). Mater. Res. Bull. 46, 1467-1472.

Liu, L., Zheng, S., Huang, R., Shi, D., Huang, Y., Wu, S., Li, Y., Fang, L. \& Hu, C. (2013). Adv. Powder Technol. 24, 908-912.

Ma, C. \& Tan, X. (2010). Solid State Commun. 150, 1497-1500.

Ma, C. \& Tan, X. (2011). J. Am. Ceram. Soc. 94, 4040-4044.

Ma, C., Tan, X., Dul'kin, E. \& Roth, M. (2010). J. Appl. Phys. 108, 104105.

Mgbemere, H. E., Hinterstein, M. \& Schneider, G. A. (2012). J. Eur. Ceram. Soc. 32, 4341-4352.

Miehe, G. (2002). Program for Interpreting Electron Diffraction Patterns (PIEP). Version 7.12. Institute for Materials Science, Darmstadt University of Technology, Germany.

Noheda, B., Cox, D. E., Shirane, G., Gonzalo, J. A., Cross, L. E. \& Park, S.-E. (1999). Appl. Phys. Lett. 74, 2059.

Noheda, B., Gonzalo, J. A., Cross, L. E., Guo, R., Park, S. E., Cox, D. E. \& Shirane, G. (2000). Phys. Rev. B, 61, 8687-8695.

Panda, P. K. (2009). J. Mater. Sci. 44, 5049-5062.
Ranjan, R., Garg, R., Kothai, V., Agrawal, A., Senyshyn, A. \& Boysen, H. (2010). J. Phys. Condens. Matter, 22, 075901.

Rao, B. N., Fitch, A. N. \& Ranjan, R. (2013). Phys. Rev. B, 87, 060102.

Rödel, J., Jo, W., Seifert, K. T. P., Anton, E.-M., Granzow, T. \& Damjanovic, D. (2009). J. Am. Ceram. Soc. 92, 1153-1177.

Roisnel, T. \& Rodriguez-Carvajal, J. (2001). Mater. Sci. Forum, 118, 378-381.

Schmitt, L. A., Hinterstein, M., Kleebe, H.-J. \& Fuess, H. (2010). J. Appl. Cryst. 43, 805-810.

Schmitt, L. A. \& Kleebe, H.-J. (2010). Funct. Mater. Lett. 03, 55-58.

Schütz, D., Deluca, M., Krauss, W., Feteira, A., Jackson, T. \& Reichmann, K. (2012). Adv. Funct. Mater. 22, 2285-2294.

Shrout, T. R. \& Zhang, S. J. (2007). J. Electroceram. 19, 185.

Singh, K., Lingwal, V., Bhatt, S. C., Panwar, N. S. \& Semwal, B. S. (2001). Mater. Res. Bull. 36, 2365-2374.

Stephens, P. W. (1999). J. Appl. Cryst. 32, 281-289.

Takenaka, T., Nagata, H. \& Hiruma, Y. (2008). Jpn. J. Appl. Phys. 47, 3787-3801.

Tellier, J., Malic, B., Dkhil, B., Jenko, D., Cilensek, J. \& Kosec, M. (2009). Solid State Sci. 11, 320-324.

Thompson, P., Cox, D. E. \& Hastings, J. B. (1987). J. Appl. Cryst. 20, 79-83.

Trolliard, G. \& Dorcet, V. (2008). Chem. Mater. 20, 5074-5082.

Usher, T.-M., Forrester, J. S., dela Cruz, C. R. \& Jones, J. L. (2012). Appl. Phys. Lett. 101, 152906.

Wang, R., Bando, H. \& Itoh, M. (2009). Appl. Phys. Lett. 95, 092905.

Woodward, D. I. \& Reaney, I. M. (2005). Acta Cryst. B61, 387-399.

Zhang, S., Xia, R. \& Shrout, T. R. (2007). Appl. Phys. Lett. 91, 132913.

Zuo, R., Fang, X. \& Ye, C. (2007). Appl. Phys. Lett. 90, 092904. 